\title{
Determinants of aggregate length of hospital stay in the last year of life in Switzerland
}

Damian Hedinger ${ }^{1}$, Julia Braun${ }^{1}$, Vladimir Kaplan², Matthias Bopp ${ }^{1 *}$ and for the Swiss National Cohort Study Group

\begin{abstract}
Background: In contrast to individual preferences, most people in developed countries die in health care institutions, with a considerable impact on health care resource use and costs. However, evidence about determinants of aggregate length of hospital stay in the last year preceding death is scant.
\end{abstract}

Methods: Nationwide individual patient data from Swiss hospital discharge statistics were linked with census and mortality records from the Swiss National Cohort. We explored determinants of aggregate length of hospital stay in the last year of life in N=35,598 inpatients $\geq 65$ years who deceased in 2007 or 2008.

Results: The average aggregate length of hospital stay in the last year of life was substantially longer in the German speaking region compared to the French (IRR 1.36 [95 \% Cl 1.32-1.40]) and Italian (IRR 1.22 [95 \% Cl 1.16-1. 29]) speaking region of the country. Increasing age, female sex, multimorbidity, being divorced, foreign nationality, and high educational level prolonged, whereas home ownership shortened the aggregate length of hospital stay. Individuals with complementary private health insurance plans had longer stays than those with compulsory health insurance plans (IRR 1.04 [95 \% Cl 1.01-1.07]).

Conclusions: The aggregate length of hospital stay during the last year of life was substantially determined by regional and socio-demographic characteristics, and only partially explained by differential health conditions. Therefore, more detailed studies need to evaluate, whether these differences are based on patients' health care needs and preferences, or whether they are supply-driven.

Keywords: Inpatient care, Length of hospital stay, End-of-life, Socio-demographic determinants, ICU, Regional variation

Abbreviations: BIC, Bayesian information criterion; COPD, Chronic obstructive pulmonary disease; IRR, Incidence rate ratio; ISCED, International Standard Classification of Education; MedStat, Medical statistics of the Swiss hospitals; SNC, Swiss National Cohort

\section{Background}

One of the major concerns relating to demographic aging is the increasing use of health care resources by the growing number of elderly ( $\geq 65$ years) and oldestold ( $\geq 80$ years) patients. Survival and longevity have increased in several chronic conditions due to modern medical care [1]. However, there is evidence that chronic medical conditions and their multiples (multimorbidity)

\footnotetext{
* Correspondence: Matthias.Bopp@uzh.ch

'Epidemiology, Biostatistics and Prevention Institute, University of Zurich, Hirschengraben 84, CH-8001 Zürich, Switzerland

Full list of author information is available at the end of the article
}

increase with age and before death [2]. Nowadays, the majority of people in industrialised countries die in health care institutions such as hospitals and nursing homes. However, there is a large variation in the proportion of deaths occurring in hospitals [3]. In Europe, these differences are often explained by the differing availability of alternative health care services for elderly and oldest-old patients (i.e., nursing homes) [4].

Previous research related to health care resource use has shown that the average cost of hospital care significantly increased with age. However, it is argued that not age per se is the main driver of health care costs, but 
ultimate closeness to death $[5,6]$. Nevertheless, the place of death has a considerable impact on health care resource use, since end-of-life care is generally more expensive in hospitals than in nursing homes, and even much more expensive than outpatient care at home [7, 8]. Studies from the US and the Netherlands have shown that the last-year-of-life health expenditures constituted between 10 and $25 \%$ of all medical expenses during life $[6,8,9]$. There is evidence that these costs may even increase in the future.[10]. In Switzerland, health insurance has been compulsory since 1996 and covers, in principle, all medical treatments and diagnostics prescribed by doctors. It also covers the costs of medical care provided in longterm care institutions [11]. Patients contribute to the costs of care through co-insurance rates up to an annual ceiling and a modest flat daily co-payment for hospital stays. Three out of ten people have a private supplementary health insurance typically covering private rooms in hospitals. Switzerland-specific information concerning the costs of hospital stays in the last year of life is lacking. There is, however, no doubt that hospital inpatient stays are more expensive than any other kind of health care and therefore offer a large potential for cost saving.

A difficult clinical challenge is the admission of oldestold patients to an intensive care unit (ICU). ICU care is expensive, highly rationed, morally charged, and therefore, decisions regarding admissions to ICUs remain crucial questions in oldest-old patients [12]. There is evidence that admission rates to acute care hospitals as place of death decreased, while ICU admissions in the last month of life increased [13]. In addition, huge differences regarding ICU admission rates and regarding physicians' opinions of appropriateness of ICU care were observed between hospitals [13, 14]. Furthermore, adequate support for shared decision-making and patientcare specific measures can increase the family satisfaction with ICU care and therefore better meet the patient's and his/her family's preferences of end-of-life care [15].

It is well known that health care use depends not only on age and proximity to death, but also on other sociodemographic factors like gender, educational level, and marital status [16]. Educational differences regarding morbidity and mortality are an uncontested finding in social epidemiology [17-19]. There is also evidence that socio-demographic determinants have a significant influence on the place of death $[20,21]$. Furthermore, there is solid evidence of regional differences in health care use and place of death [7, 22, 23]. However, to our knowledge, evidence on determinants of frequency and length of hospital stay in the time period preceding death is sparse.

We therefore explored, based on linked census and hospital discharge statistics, the potential impact of medical (e.g. multimorbidity) and social determinants (e.g., education, home ownership, marital status) on the aggregate length of hospital stay in the last year of life among those deceased in hospitals.

\section{Methods \\ Data}

We extracted data from two different sources covering all individuals living in Switzerland:

(1) The Swiss National Cohort (SNC, www.swissnationalcohort.ch) is an anonymous linkage of census, mortality and emigration records [24]. We used individual data from the 2000 census and mortality data (incl. cause of death) from 2007-08.

(2) The Swiss hospital discharge statistics (MedStat) administered by the Swiss Federal Statistical Office [25] encompasses individual information about diagnoses, treatments, discharge dates, and - crucial for record linkage - for those deceased in hospitals, dates of birth. We used data for the year 2002-2008.

Both data sources encompass the entire Swiss population and are fully anonymized. For reasons of data protection and confidentiality, there is no personal identifier that allows direct linkage of data sources on an individual level. The linkage had to rely on common identification variables such as place of residence, date of birth, and date of death. As a consequence, the linkage to the SNC was possible only for those who died in an institution. The detailed linkage process is described elsewhere [20]. We restricted our analyses to individuals $\geq 65$ years (i.e., born before 1942) who died in 2007 and 2008 in hospital settings. As we know from a place of death study in Switzerland [26], about $45 \%$ of men and $35 \%$ of women above 65 years die in hospitals. In the study period, almost $99 \%$ of deaths in hospitals could be linked with the SNC and are therefore accounted for in this analysis.

\section{Study design}

Our outcome variable was the aggregate number of days spent in a hospital during the last year (365 days) of life. The independent variables were grouped into medical, individual, familial/housing, and structural/regional attributes. As control variables we included age (at the time of death) and sex. Cause of death was categorized as: malignant neoplasms (ICD 10: C00-C99), coronary heart disease (I20-I25), stroke (I60-I69), chronic obstructive pulmonary disease (COPD, J40-J47), dementia (F01, F03, G30), and all other causes. From MEDSTAT, we derived information about multimorbidity ( $\geq 2$ chronic conditions), assessed from inpatient diagnoses within 2-6 years before death. We defined chronic conditions using 
ICPC-2, of which 129 rubrics were classified as chronic conditions [27]. We used specific time windows in order to test the different impact of health indicators which are closer to or more distant from death. For better understanding of our health variables, Fig. 1 presents examples of two persons with corresponding time windows. In addition, we generated an ICU variable to identify the influence of ICU care during the last hospital stay: no ICU care, ICU care $1-48 \mathrm{~h}$, or more than $48 \mathrm{~h}$. From the 2000 census data, we derived the educational level according to the International Standard Classification of Education (ISCED), version 1997: no or low secondary education (ISCED 0-2), post-secondary non-tertiary ("medium", ISCED 3-4), and tertiary education ("high", ISCED 5). From the 2000 census we extracted information about home ownership (owner-occupier household). From MEDSTAT, we extracted the hospital room category of the respective hospitalisation (private/semi-private or regular). As very few patients upgrade their hospital room category from regular to private/semi-private, since they have to reimburse additional costs, the hospital room category is representative for the health insurance plan (private/compulsory). Marital status was derived from the SNC and assessed at the time of death (never married, married, widowed and divorced). Place of residence was categorized into the three main language areas in Switzerland, namely the German, French and Italian speaking part. The nationality was derived from the SNC and categorized as Swiss or foreign. To account for geographical variation in nursing home bed availability, we included the nursing home bed density per 100 inhabitants 65 years or older derived from the SNC.

\section{Statistical methods}

For descriptive analyses, we calculated means, frequencies and proportions of the respective variables. To detect a right-skewed distribution, we report means, medians, SD and $25^{\text {th }}$ and $75^{\text {th }}$ percentiles of our outcomes. The outcome variable "aggregate number of days spent in a hospital in the last year of life" is a count variable. Therefore, a count regression such as Poisson or negative binomial is appropriate for statistical modelling. We chose the negative binomial model over the Poisson model to account for the strong over dispersion in the outcome variable. We also considered to use a zeroinflated negative binomial model. However, the number of zeros in our data set is not extremely large (less than $5 \%$ of the study population) and there is no reason to believe that part of the patients differs from the others in that they per se cannot have any counts but zero. Goodness-of-fit criteria like AIC and BIC performed slightly worse in the zero-inflated model than in the regular binomial model and also the Vuong test showed a non-significant result with a p-value close to 1 . For these reasons, we preferred a regular negative binomial model. We calculated incident rate ratios (IRR) [28] to assess the impact of several independent variables on the number of days spent in a hospital in the last year of life. For better understanding of the interpretation of IRR, we give a reading example using the estimated IRR of

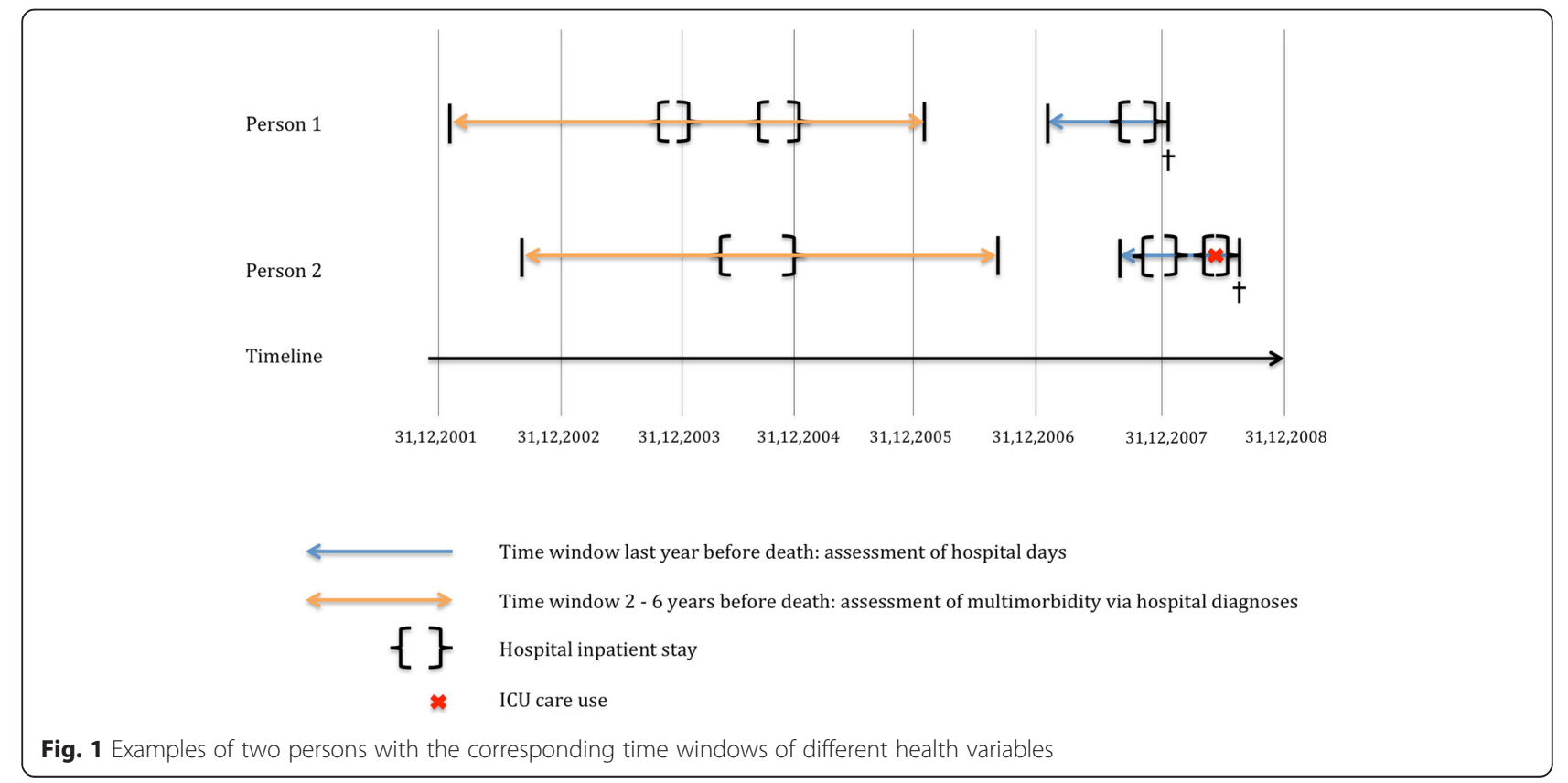


1.05 for a person with "high" educational level (cf. Table 2): Compared to a person with "medium" educational level (reference category), the expected aggregate number of days spent in a hospital during the last year of life was for a person with "high" educational level 1.05 times as high as in the reference category. We used likelihood ratio tests to calculate joint p-values of categorical variables. The level of significance was set to $\alpha=0.05$ (two-sided). To improve the fit of the model, we explored relevant interaction effects between sex and marital status, cause of death and ICU use, and multimorbidity and ICU use. However, none of these interaction terms improved the model with respect to the Bayesian information criterion (BIC), and for this reason, they were not included in the final model. Due to substantial differences between the language regions, we performed also a stratified analysis for the two major language regions (German vs. French speaking part) of Switzerland. In addition, we also checked for the above mentioned interaction effects in each of the models, but again, with respect to the $\mathrm{BIC}$, the interaction terms didn't improve the models.

\section{Results}

We identified $N=35,598$ patients $(18,993$ men and 16,605 women) $\geq 65$ years who died in 2007 and 2008 in hospital settings. Table 1 presents the characteristics of our study population.

We observed a strong right-skewed deviation of the distribution of aggregate number of days spent in a hospital during the last year of life. Less than one out of five patients received ICU care during the terminal hospital stay.

Aggregate length of hospital stay during the last year of life (mean, SD) and estimated incidence rate ratios (IRR) for baseline characteristics are presented in Table 2. A stratified analysis for the German and French speaking part of the country is given in Appendix 1 and 2 .

Increasing age was a significant predictor for shorter aggregate length of hospital stay in the last year of life. There were also significant differences in aggregate length of stay with respect to specific causes of death: compared to patients dying of cancer, those dying of coronary heart disease, stroke, and chronic obstructive pulmonary disease had a significantly shorter, while those dying of dementia significantly longer length of hospital stay. Multimorbidity significantly increased the aggregate duration of hospital stay. For those without any hospital admission within 2-6 years before death (and consequently no information about multimorbidity), the aggregate time spent in hospitals during the last year of life was significantly shorter than for those previously hospitalized but without a diagnosis of multimorbidity. For those who received ICU care for $\leq 48 \mathrm{~h}$ during the last hospitalisation, aggregate length of stay during the last year of life was significantly shorter than for those who received no ICU care or ICU care for $>48 \mathrm{~h}$. There was a remarkable difference between the French and the German speaking part regarding those who received ICU for $>48 \mathrm{~h}$ (Appendix Tables 1 and 2): While this group had a significantly longer mean aggregate length of stay in the German speaking part, we found the opposite effect in the French speaking part. Patients with a high or unknown educational level had significantly longer aggregate stays than those with a medium or low educational level. Home ownership was a significant predictor of shorter aggregate length of stay. Those hospitalized in a semi-private/private room category (proxy for a private health insurance plan) had a significant longer aggregate stay than those hospitalized in a regular room category (proxy for a compulsory health insurance plan). Divorced patients had significantly longer aggregate stays compared to those married, widowed, and never married. Swiss citizens had significantly shorter aggregate stays than foreigners. Compared to the German speaking region, the aggregate length of hospital stay in the last year of life was longer in the Italian and even more so in the French speaking part of Switzerland. We also found a significant effect of nursing bed density on the aggregate length of stay: high regional nursing bed density was associated with a shorter aggregate length of hospital stays during the last year of life.

\section{Discussion}

As expected, health-related characteristics such as specific cause of death, multimorbidity, and admission to intensive care during the last hospitalization had a significant impact on the aggregate length of hospital stays during the last year of life. However, the duration was not dependent on health-related characteristics alone, but also on a variety of social determinants such as educational level, home ownership, hospital room category (proxy of the health insurance plan), language region, and nursing bed density.

A noteworthy observation was the substantial variation between aggregate length of hospital stays during the last year of life in the different language regions of Switzerland. Patients living in the Italian, and particularly those living in the French part spent more days in hospitals during the last year of life than those living in the German part. Prior studies gave already evidence for regional variation in health care use in Switzerland and in the US [7, 22]. Consistently, a recent study from Switzerland found that people living in the French or Italian part were more likely to die in an institution than those living in the German part [20]. One could argue that this might point to a higher propensity for more 
Table 1 Baseline characteristics of patients deceased in hospitals in 2007 and 2008 ( $N=35,598)$

\begin{tabular}{ll}
\hline Aggregate length of hospital stay in the last year of life (days) & \\
\hline Length of hospital stays last 365 days, mean & 32.1 \\
Length of hospital stays last 365 days, median & 20 \\
Length of hospital stays last 365 days, $25^{\text {th }}$ percentile & 7 \\
Length of hospital stays last 365 days, $75^{\text {th }}$ percentile & 44 \\
Length of hospital stays last 365 days, SD & 38 \\
Mean age at time of death (years) & 80.8
\end{tabular}

Sex

men

women

Cause of death

cancer

coronary heart disease

stroke

chronic obstructive pulmonary disease

dementia

other

Multimorbidity (assessed in time window 2-6 years before death)

no

yes

unknown (no hospital admission)

Intensive care during last hospital stay (ICU)

none

$1-48 \mathrm{~h}$

more than $48 \mathrm{~h}$

Educational level

low

medium

high

unknown

House ownership

owner-occupier

tenant

Room category

regular

semi private/private

Marital status

never married

married

widowed

divorced

Nationality

Swiss

foreign
$53.4 \%$

$46.6 \%$

$35.5 \%$

$12.5 \%$

$8.0 \%$

$3.3 \%$

$1.3 \%$

$39.4 \%$

$23.2 \%$

$34.2 \%$

$42.7 \%$

$83.2 \%$

$8.2 \%$

$8.6 \%$

$38.3 \%$

$34.9 \%$

$11.3 \%$

$15.6 \%$

$43.2 \%$

$56.8 \%$

$77.9 \%$

$22.1 \%$

$8.0 \%$

$50.4 \%$

$34.5 \%$

$7.1 \%$

$92.2 \%$

$7.8 \%$
Table 1 Baseline characteristics of patients deceased in hospitals in 2007 and $2008(N=35,598)$ (Continued)

\begin{tabular}{lc}
\hline $\begin{array}{l}\text { Language region } \\
\text { German }\end{array}$ & $67.5 \%$ \\
French & $27.4 \%$ \\
Italian & $5.1 \%$ \\
Nursing home bed density & 6.7 \\
\hline * average number of nursing home beds per 100 habitants aged 65 years \\
$\begin{array}{l}\text { and older (per } 106 \text { regions) } \\
\text { Data source: Swiss Federal Statistical Office, MedStat, SNC }\end{array}$
\end{tabular}

aggressive (and possibly futile) care at the end-of-life due to cultural differences between the language regions [29].

The well-known divergence between the preferred and actual place of death might be due to unmet wishes of patients and their families regarding hospital and longterm care admissions before death and might represent an indicator of a low quality of dying [30-32]. Therefore, home-based palliative care models, which decrease the time spent in hospitals during the last months of life, may increase the quality of dying and end-of-life care $[33,34]$. Different palliative care models have been developed in some European countries to implement alternative ways and increase the quality of end-of-life care, however, their effectiveness and impact on care before death varies substantially [35]. A successful integration of palliative care services in the ICU can increase the quality, save costs and improve patient and family satisfaction [36]. Unfortunately, due to regional variation and lack of reliable data, an evaluation of palliative care in Switzerland is difficult [37].

Another remarkable observation is the variation of ICU care during the terminal hospitalization before death. In the US, around $20 \%$ of deaths occur in ICU settings [9] despite questionable benefits regarding survival and quality of life [14]. Therefore, ICU care offers an important potential for health costs saving [9]. In our study population, $16.8 \%$ of all patients received ICU care during the terminal hospitalization. Many of those with an ICU stay $\leq 48 \mathrm{~h}$ died shortly after admission. Such a "sudden death" was associated with a significant shorter length of aggregate hospital stay in the last year of life. However, regarding aggregate length of hospital stay in the last year of life, those with an ICU stay $>48 \mathrm{~h}$ did not significantly differ from those receiving no ICU care at all.

Multimorbidity was another informative determinant of aggregate length of hospital stay during the last year of life: Multimorbidity diagnosed during hospitalizations within 2-6 years before death was associated with a longer aggregate length of hospital stay in the last year of life, probably due to worse health status and therefore more medical needs of these often very sick patients. 
Table 2 Mean aggregate length and results of the negative binomial regression analysis $(N=35,598)$

\begin{tabular}{|c|c|c|c|c|}
\hline & \multicolumn{2}{|c|}{$\begin{array}{l}\text { Length of stay } \\
\text { (days) }\end{array}$} & \multirow[t]{2}{*}{ IRR } & \multirow[t]{2}{*}{$95 \% \mathrm{Cl}$} \\
\hline & Mean & SD & & \\
\hline Age (at time of death) $(p<0.001)$ & & & 0.98 & $0.98-0.99$ \\
\hline \multicolumn{5}{|l|}{$\operatorname{Sex}(p<0.05)$} \\
\hline men & 32.3 & 38.7 & 1.00 & \\
\hline women & 31.9 & 38.2 & 1.03 & $1.00-1.06$ \\
\hline \multicolumn{5}{|l|}{ Cause of death $(p<0.001)$} \\
\hline cancer (ref.) & 39.1 & 36.4 & 1.00 & \\
\hline coronary heart disease & 23.6 & 34.8 & 0.68 & $0.65-0.70$ \\
\hline stroke & 23.2 & 35.6 & 0.65 & $0.62-0.68$ \\
\hline COPD & 35.6 & 44.3 & 0.89 & $0.83-0.95$ \\
\hline dementia & 61.2 & 72.6 & 1.74 & $1.57-1.92$ \\
\hline other & 29.0 & 38.3 & 0.81 & $0.78-0.83$ \\
\hline \multicolumn{5}{|l|}{ Multimorbidity $(p<0.001)$} \\
\hline no (ref.) & 33.9 & 39.7 & 1.00 & \\
\hline yes & 36.6 & 41.3 & 1.07 & $1.04-1.10$ \\
\hline unknown (no hospital stay) & 27.5 & 34.8 & 0.79 & $0.77-0.82$ \\
\hline \multicolumn{5}{|l|}{ ICU during last stay $(p<0.001)$} \\
\hline no (ref.) & 33.3 & 39.5 & 1.00 & \\
\hline $1-48 \mathrm{~h}$ & 20.3 & 29.9 & 0.65 & $0.62-0.67$ \\
\hline more than $48 \mathrm{~h}$ & 31.3 & 33.2 & 0.97 & $0.93-1.01$ \\
\hline \multicolumn{5}{|l|}{ Educational level $(p<0.001)$} \\
\hline medium (ref.) & 31.3 & 36.1 & 1.00 & \\
\hline low & 31.3 & 37.6 & 0.98 & $0.95-1.01$ \\
\hline high & 34.4 & 41.5 & 1.05 & $1.01-1.09$ \\
\hline unknown & 34.0 & 43.2 & 1.06 & $1.02-1.10$ \\
\hline \multicolumn{5}{|l|}{ House or flat owner $(p<0.001)$} \\
\hline tenant (ref.) & 33.1 & 40.0 & 1.00 & \\
\hline owner-occupier & 30.7 & 36.4 & 0.93 & $0.91-0.95$ \\
\hline \multicolumn{5}{|l|}{ Room category $(p<0.01)$} \\
\hline regular (ref.) & 32.4 & 40.1 & 1.00 & \\
\hline semi-private/private & 31.1 & 32.2 & 1.04 & $1.01-1.07$ \\
\hline \multicolumn{5}{|l|}{ Marital status $(p<0.001)$} \\
\hline married (ref.) & 32.4 & 37.1 & 1.00 & \\
\hline never married & 33.1 & 42.9 & 1.04 & $0.99-1.08$ \\
\hline widowed & 30.0 & 37.9 & 1.03 & $1.00-1.06$ \\
\hline divorced & 38.4 & 44.9 & 1.12 & $1.07-1.17$ \\
\hline \multicolumn{5}{|l|}{ Nationality $(p<0.001)$} \\
\hline Swiss (ref.) & 31.4 & 37.8 & 1.00 & \\
\hline foreign & 39.8 & 45.1 & 1.09 & $1.04-1.14$ \\
\hline \multicolumn{5}{|l|}{ Language region $(p<0.001)$} \\
\hline German (ref.) & 27.6 & 34.1 & 1.00 & \\
\hline French & 42.1 & 46.5 & 1.36 & $1.32-1.40$ \\
\hline Italian & 37.3 & 34.8 & 1.22 & $1.16-1.29$ \\
\hline
\end{tabular}

Table 2 Mean aggregate length and results of the negative binomial regression analysis $(N=35,598)$ (Continued)

\begin{tabular}{lll}
\hline Nursing home bed density ${ }^{\mathrm{a}}(p>0.001)$ & 0.96 & $0.95-0.97$
\end{tabular}

IRR incidence rate ratios, $95 \% \mathrm{Cl}=95 \%$ confidence interval, $p$-values from likelihood ratio tests

${ }^{\mathrm{a}}=$ average number of nursing home beds per 100 habitants aged 65 years and older (per 106 regions)

Data source: Swiss Federal Statistical Office, MedStat, SNC

Conversely, patients without hospitalization within 2-6 years before death (and therefore no information on multimorbidity) had substantially shorter aggregate hospital stays and received less medical care.

We also found evidence for effects of socio-economic differences on the aggregate length of hospital stays. However, compared to other studies on morbidity and mortality differences in the old age [17-19], our results were less consistent. Higher educational level was associated with increased aggregate length of stay. Conversely, home ownership, another proxy for higher socioeconomic position, was associated with a significantly shorter aggregate length of stays. Patients with presumably better health insurance plans (semi-private/private room category) had longer stays compared to those with regular coverage plans. A similar study from Finland showed that educational level had little effect on hospital care in the last 7 years preceding death [16]. Conversely, socio-economic characteristics were important determinants of admission to and death in a nursing home but not of acute care hospital length of stay [16, 20]. Finally, regional attributes like language region and nursing home bed density were more consistent predictors of aggregate length of hospital stays in the last year of life compared to socio-economic determinants. As research on differences in health care between the two major language regions in Switzerland is scarce, it is difficult to elucidate our results. We found evidence for cultural differences in hospital treatments before death, because longer hospital stays, which imply more aggressive treatments before death, were more common in the French and Italian speaking part of Switzerland compared to the German speaking part. This result is in line with higher odds of hospitals as place of death in those regions [26]. On the other side, nursing home bed density - known to be lower in the French speaking part [38] - had a higher negative impact on length of hospital stays in this region, suggesting an important role of supply-sensitive care.

\section{Strengths and limitations}

One major strength of our study is the national coverage and the size of the study population. Another strength is the uniqueness of the data base generated by linking census with mortality and other 
administrative data. Furthermore, our study gives empirical evidence for several health indicators considering an extended time period before, and therefore little related to death.

Besides such strengths, the study has some limitations: As usual for secondary data and particularly administrative data analyses, by far not all desirable information is available (e.g., information about palliative care) and the use of proxy variables with limited validity is inevitable. However, the use of such weak measures does not necessarily lead to a systematic information bias but rather results in a non-differential misclassification and therefore underestimation of the true association. In Switzerland, there is no statistical information on the outpatient health care. Therefore it was not possible to assess diagnostic information of people that have not been hospitalized during the study period. As we restricted the study population to people having died in hospitals, our study population is not representative for the general population above 65 years. This is the reason why some causes of death (e.g. dementia) have only small percentages. Another limitation of the study is the incomplete linkage of all deaths to a census record as well as to a respective hospital record.

The percentage of hospital deaths is in line with another study from Switzerland [39]. Our study is based on a virtually complete nationwide assessment of hospitalizations in 2002-2008 and thus mirrors the situation in Switzerland at that time. But due to differences in methodology, study populations and health care systems, we cannot validly compare our results with those of other countries.

\section{Conclusions}

The aggregate time spent in hospitals in the last year of life is mainly determined by differential health conditions and therefore, differential needs of medical care. However, remarkable unexplained disparities between the German and the Latin (French and Italian) speaking region of Switzerland remain: frequent and/ or longer hospital stays in the last year of life are more common in the French and Italian speaking part of the country, probably due to cultural differences. Therefore, more detailed studies need to evaluate, whether these differences are based on patients' health care needs and preferences, or whether they are supply-driven. As hospital care in general and ICU care in particular is expensive and often unwanted by those deceasing, more efforts to minimize aggressive care at the end-of-life - especially in the Latin regions of Switzerland - may be a promising target of health policy.

\section{Appendix}

Table 3 Results for the German speaking part of Switzerland $(N=24,041)$

\begin{tabular}{|c|c|c|c|c|}
\hline & \multicolumn{2}{|c|}{ Length of stay (days) } & \multirow[t]{2}{*}{ IRR } & \multirow[t]{2}{*}{$95 \% \mathrm{Cl}$} \\
\hline & Mean & SD & & \\
\hline Age (at time of death) $(p<0.001)$ & & & 0.98 & $0.98-0.98$ \\
\hline \multicolumn{5}{|l|}{$\operatorname{Sex}(p<0.05)$} \\
\hline men & 27.9 & 35.0 & 1.00 & \\
\hline women & 27.4 & 33.8 & 1.04 & $1.00-1.07$ \\
\hline \multicolumn{5}{|l|}{ Cause of death $(p<0.001)$} \\
\hline cancer (ref.) & 34.1 & 32.5 & 1.00 & \\
\hline coronary heart disease & 21.4 & 31.8 & 0.68 & $0.65-0.72$ \\
\hline stroke & 19.6 & 30.4 & 0.63 & $0.60-0.67$ \\
\hline COPD & 29.7 & 36.7 & 0.85 & $0.79-1.92$ \\
\hline dementia & 57.7 & 74.6 & 1.96 & $1.71-2.25$ \\
\hline other & 25.0 & 33.5 & 0.80 & $0.77-0.82$ \\
\hline \multicolumn{5}{|l|}{ Multimorbidity $(p<0.001)$} \\
\hline no (ref.) & 31.1 & 38.3 & 1.00 & \\
\hline yes & 30.5 & 34.4 & 1.01 & $0.98-1.05$ \\
\hline unknown (no hospital stay) & 23.8 & 30.9 & 0.75 & $0.73-0.78$ \\
\hline \multicolumn{5}{|l|}{ ICU during last stay $(p<0.001)$} \\
\hline no (ref.) & 28.3 & 34.8 & 1.00 & \\
\hline $1-48 \mathrm{~h}$ & 19.0 & 27.9 & 0.69 & $0.66-0.73$ \\
\hline more than $48 \mathrm{~h}$ & 29.8 & 31.3 & 1.07 & $1.01-1.13$ \\
\hline \multicolumn{5}{|l|}{ Educational level $(p<0.001)$} \\
\hline medium (ref.) & 28.3 & 33.2 & 1.00 & \\
\hline low & 26.4 & 32.5 & 0.96 & $0.93-0.99$ \\
\hline high & 29.9 & 37.8 & 1.04 & $0.99-1.09$ \\
\hline unknown & 27.6 & 37.4 & 1.03 & $0.99-1.08$ \\
\hline \multicolumn{5}{|l|}{ House or flat owner $(p<0.001)$} \\
\hline tenant (ref.) & 28.3 & 34.6 & 1.00 & \\
\hline owner-occupier & 26.8 & 33.4 & 0.93 & $0.90-0.96$ \\
\hline \multicolumn{5}{|l|}{ Room category $(p<0.01)$} \\
\hline regular (ref.) & 26.9 & 35.0 & 1.00 & \\
\hline semi-private/private & 29.7 & 31.3 & 1.08 & $1.04-1.11$ \\
\hline \multicolumn{5}{|l|}{ Marital status $(p<0.001)$} \\
\hline married (ref.) & 28.5 & 33.4 & 1.00 & \\
\hline never married & 29.4 & 40.7 & 1.04 & $0.98-1.09$ \\
\hline widowed & 25.1 & 32.5 & 1.00 & $0.96-1.04$ \\
\hline divorced & 32.5 & 37.8 & 1.10 & $1.03-1.16$ \\
\hline \multicolumn{5}{|l|}{ Nationality $(p<0.001)$} \\
\hline Swiss (ref.) & 27.3 & 33.8 & 1.00 & \\
\hline foreign & 33.0 & 38.2 & 1.09 & $1.03-1.16$ \\
\hline Nursing home bed density ${ }^{a}(p>0.001)$ & & & 0.97 & $0.97-0.98$ \\
\hline
\end{tabular}

IRR incidence rate ratios, $95 \% \mathrm{Cl}=95 \%$ confidence interval, $p$-values from likelihood ratio tests

${ }^{a}=$ average number of nursing home beds per 100 habitants aged 65 years and older (per 106 regions)

Data source: Swiss Federal Statistical Office, MedStat, SNC 
Table 4 Results for the French speaking part of Switzerland $(N=9,745)$

\begin{tabular}{|c|c|c|c|c|}
\hline & \multicolumn{2}{|c|}{$\begin{array}{l}\text { Length of stay } \\
\text { (days) }\end{array}$} & \multirow[t]{2}{*}{ IRR } & \multirow[t]{2}{*}{$95 \% \mathrm{Cl}$} \\
\hline & Mean & SD & & \\
\hline Age (at time of death) $(p<0.001)$ & & & 0.99 & $0.99-0.99$ \\
\hline \multicolumn{5}{|l|}{$\operatorname{Sex}(p<0.05)$} \\
\hline men & 41.6 & 46.5 & 1.00 & \\
\hline women & 42.6 & 46.5 & 1.01 & $0.97-1.06$ \\
\hline \multicolumn{5}{|l|}{ Cause of death $(p<0.001)$} \\
\hline cancer (ref.) & 48.1 & 42.1 & 1.00 & \\
\hline coronary heart disease & 30.7 & 43.4 & 0.67 & $0.62-0.73$ \\
\hline stroke & 32.8 & 46.6 & 0.70 & $0.65-0.77$ \\
\hline COPD & 49.8 & 58.1 & 0.96 & $0.85-1.09$ \\
\hline dementia & 70.0 & 72.3 & 1.54 & $1.31-1.82$ \\
\hline other & 38.5 & 47.5 & 0.84 & $0.80-0.89$ \\
\hline \multicolumn{5}{|l|}{ Multimorbidity $(p<0.001)$} \\
\hline no (ref.) & 40.6 & 43.0 & 1.00 & \\
\hline yes & 48.0 & 51.0 & 1.22 & $1.15-1.30$ \\
\hline unknown (no hospital stay) & 36.8 & 42.6 & 0.91 & $0.86-0.96$ \\
\hline \multicolumn{5}{|l|}{ ICU during last stay $(p<0.001)$} \\
\hline no (ref.) & 44.5 & 47.7 & 1.00 & \\
\hline $1-48 \mathrm{~h}$ & 23.8 & 35.7 & 0.54 & $0.49-0.58$ \\
\hline more than $48 \mathrm{~h}$ & 33.9 & 37.5 & 0.76 & $0.71-0.82$ \\
\hline \multicolumn{5}{|l|}{ Educational level $(p<0.001)$} \\
\hline medium (ref.) & 40.5 & 43.6 & 1.00 & \\
\hline low & 41.2 & 48.9 & 1.02 & $0.96-1.07$ \\
\hline high & 43.9 & 48.0 & 1.09 & $1.02-1.17$ \\
\hline unknown & 44.7 & 50.4 & 1.09 & $1.03-1.16$ \\
\hline \multicolumn{5}{|l|}{ House or flat owner $(p<0.001)$} \\
\hline tenant (ref.) & 44.1 & 49.2 & 1.00 & \\
\hline owner-occupier & 39.2 & 42.2 & 0.92 & $0.88-0.97$ \\
\hline \multicolumn{5}{|l|}{ Room category $(p<0.01)$} \\
\hline regular (ref.) & 42.9 & 47.6 & 1.00 & \\
\hline semi-private/private & 35.0 & 34.5 & 0.83 & $0.77-0.89$ \\
\hline \multicolumn{5}{|l|}{ Marital status $(p<0.001)$} \\
\hline married (ref.) & 40.9 & 43.9 & 1.00 & \\
\hline never married & 41.8 & 48.5 & 1.02 & $0.94-1.11$ \\
\hline widowed & 42.0 & 47.5 & 1.08 & $1.02-1.14$ \\
\hline divorced & 50.0 & 55.3 & 1.15 & $1.06-1.25$ \\
\hline \multicolumn{5}{|l|}{ Nationality $(p<0.001)$} \\
\hline Swiss (ref.) & 41.1 & 45.5 & 1.00 & \\
\hline foreign & 49.9 & 53.5 & 1.11 & $1.04-1.19$ \\
\hline Nursing home bed density ${ }^{a}(p>0.001)$ & & & 0.89 & $0.88-0.91$ \\
\hline
\end{tabular}

IRR incidence rate ratios, $95 \% \mathrm{Cl}=95 \%$ confidence interval, $p$-values from likelihood ratio tests

${ }^{\mathrm{a}}=$ average number of nursing home beds per 100 habitants aged 65 years and older (per 106 regions)

Data source: Swiss Federal Statistical Office, MedStat, SNC

\section{Acknowledgements}

We thank the Swiss Federal Statistical Office for providing mortality and census data, and for the support, which made the construction of the Swiss National Cohort and this study possible. The members of the Swiss National Cohort Study Group are Matthias Egger (Chairman of the Executive Board), Adrian Spoerri and Marcel Zwahlen (all Bern), Milo Puhan (Chairman of the Scientific Board), Matthias Bopp (both Zurich), Nino Künzli (Basel), Fred Paccaud (Lausanne), and Michel Oris (Geneva).

\section{Funding}

This study and two of the authors (DH, JB), respectively, were supported by the Swiss National Science Foundation (grants 406740 139331,

33CS30_134273 and 33CS30_148415). The funding organisation had no influence in study design, analysis or interpretation of data.

\section{Availability of data and materials}

Anonymized individual data from different data sets were used for the construction of the Swiss National Cohort (SNC). All these data are the property of the Swiss Federal Statistical Office (SFSO) and can only be made available by legal agreements with the SFSO. This also applies to derivatives such as the analysis files used for this study. However, after approval of the SNC Scientific Board, a specific SNC module contract with SFSO would allow researchers to receive analysis files for replication of the analysis. Data requests should be sent to Prof. Milo Puhan (chairman of the SNC Scientific Board, miloalan.puhan@uzh.ch).

\section{Authors' contributions}

$\mathrm{DH}$ wrote the first draft of the manuscript and conducted the statistical analyses. MB conceived and supervised the study. JB gave important statistical support for the review. VK contributed important intellectual content. All authors repeatedly edited the manuscript and approved the final version.

\section{Competing interests}

The authors declare that they have no competing interests.

\section{Consent for publication}

Not applicable.

Ethics approval and consent to participate

Ethics approval for this study was given by the ethics committee of the Canton of Zurich (Amendment to Stv-Nr. 13/06).

\section{Author details}

${ }^{1}$ Epidemiology, Biostatistics and Prevention Institute, University of Zurich, Hirschengraben 84, CH-8001 Zürich, Switzerland. ${ }^{2}$ Hospital of Muri, Muri, Switzerland.

Received: 4 September 2015 Accepted: 27 August 2016

Published online: 01 September 2016

\section{References}

1. Seale C. Changing patterns of death and dying. Soc Sci Med. 2000;51(6): 917-30

2. Schäfer I, von Leitner E-C, Schön G, Koller D, Hansen H, Kolonko T, et al. Multimorbidity patterns in the elderly: a new approach of disease clustering identifies complex interrelations between chronic conditions. PLoS One. 2010;5(12):e15941.

3. Broad JB, Gott M, Kim H, Boyd M, Chen H, Connolly MJ. Where do people die? An international comparison of the percentage of deaths occurring in hospital and residential aged care settings in 45 populations, using published and available statistics. Int J Public Health. 2013;58(2):257-67.

4. Cohen J, Bilsen J, Addington-Hall J, Löfmark R, Miccinesi G, Kaasa S, et al. Population-based study of dying in hospital in six European countries. Palliat Med. 2008:22(6):702-10.

5. Busse R, Krauth C, Schwartz FW. Use of acute hospital beds does not increase as the population ages: results from a seven year cohort study in Germany. J Epidemiol Community Health. 2002;56(4):289-93.

6. Polder JJ, Barendregt JJ, van Oers $\mathrm{H}$. Health care costs in the last year of life - The Dutch experience. Soc Sci Med. 2006;63:1720-31. 
7. Reich $\mathrm{O}$, Signorell A, Busato A. Place of death and health care utilization for people in the last 6 months of life in Switzerland: a retrospective analysis using administrative data. BMC Health Serv Res. 2013;13:116.

8. Hoover DR, Crystal S, Kumar R, Sambamoorthi U, Cantor JC. Medical expenditures during the last year of life: findings from the 1992-1996 medicare current beneficiary survey. Health Serv Res. 2002;37(6):1625-42

9. Curtis JR, Engelberg RA, Bensink ME, Ramsey SD. End-of-life care in the intensive care unit: can we simultaneously increase quality and reduce costs? Am J Respir Crit Care Med. 2012;186(7):587-92.

10. Wanless D. Securing our future health: taking a long-term view. final report. London: The Public Enquiry Unit; 2002.

11. OECD/WHO. OECD Reviews of Health Systems: Switzerland 2011, OECD Publishing. 2011. http://dx.doi.org/10.1787/9789264120914-en.

12. Wilson DM, Thurston A, Lichlyter B. Should the oldest-old be admitted to the intensive care unit and receive advanced life-supporting care? Crit Care Med. 2010;38(1):303-4.

13. Teno JM, Gozalo PL, Bynum JPW, Leland NE, Miller SC, Morden NE, et al. Change in end-of-life care for medicare beneficiaries. Site of death, place of care, and health care transitions in 2000, 2005, and 2009. JAMA J Am Med Assoc. 2013;309(5):470-7.

14. Boumendil A, Angus DC, Guitonneau AL, Menn AM, Ginsburg C, Takun K, et al. Variability of intensive care admission decisions for the very elderly. PLoS One. 2012;7(4):10-8.

15. Hinkle $L$, Bosslet GT, Torke AM. Factors associated with family satisfaction with End-of-life care in the ICU. Chest J. 2015;147(1):82.

16. Martikainen $P$, Murphy M, Metsä-Simola N, Häkkinen U, Moustgaard H. Seven-year hospital and nursing home care use according to age and proximity to death: variations by cause of death and socio-demographic position. J Epidemiol Community Health. 2012;66(12):1152-8.

17. Mackenbach JP, Stirbu I, Roskam A-J, Schaap MM, Menivelle G, Leinsalu M, et al. Socioeconomic inequalities in health in 22 European Countries. N Engl J Med. 2008:358:2468-81.

18. Faeh D, Bopp M. Educational inequalities in mortality and associated risk factors: German- versus French-speaking Switzerland. BMC Public Health. 2010;10:567.

19. Huisman M, Kunst AE, Bopp M, Borgan J-K, Borrell C, Costa G, et al. Educational inequalities in cause-specific mortality in middle-aged and older men and women in eight western European populations. Lancet. 2005; 365(9458):493-500.

20. Hedinger D, Braun J, Zellweger U, Kaplan V, Bopp M. Moving to and dying in a nursing home depends not only on health - An analysis of socioDemographic Determinants of Place of Death in Switzerland. PLoS One. 2014;9(11):e113236.

21. Beng AKL, Fong CW, Shum E, Goh CR, Goh KT, Chew SK. Where the elderly die: the influence of socio-demographic factors and cause of death on people dying at home. Ann Acad Med. 2009;38(8):676-83.

22. Goodman DC, Elliott FS, Chang C. After hospitalization: a Dartmouth atlas report on post-acute care for medicare beneficiaries. The Darthmouth atlas project. 2011.

23. Insam C, Paccaud F, Marques-Vidal P. The region makes the difference: disparities in management of acute myocardial infarction within Switzerland. Eur J Prev Cardiol. 2014;21:541-8.

24. Bopp M, Spoerri A, Zwahlen M, Gutzwiller F, Paccaud F, Braun-Fahrländer C, et al. Cohort profile: the Swiss National Cohort-a longitudinal study of 6.8 million people. Int J Epidemiol. 2009;38(2):379-84.

25. Swiss Federal Statistical Office. Medizinische Statistik der Krankenhäuser 2011 - Standardtabellen. Neuenburg: Swiss Federal Statistical Office; 2013.

26. Hedinger D, Haemmig O, Braun J, Bopp M. Social determinants of duration of last nursing home stay at the end of life in Switzerland: a retrospective cohort study. BMC Geriatrics 2015;15:114.

27. O'Halloran J, Miller GC, Britt H. Defining chronic conditions for primary care with ICPC-2. Fam Pract. 2004;21(4):381-6.

28. Hilbe JM. Negative binomial regression. Cambridge: Cambridge University Press; 2007.

29. Fischer S, Bosshard G, Faisst K, Tschopp A, Fischer J, Bär W, et al. Swiss doctors' attitudes towards end-of-life decisions and their determinants. A comparison of three language regions. Swiss Med Wkly. 2005;135:370-6.

30. Ishikawa Y, Fukui S, Saito T, Fujita J, Watanabe M, Yoshiuchi K. Family preference for place of death mediates the relationship between patient preference and actual place of death: a nationwide retrospective crosssectional study. PLoS One. 2013;8(3):e56848.
31. Teno JM, Clarridge BR, Casey V, Welch LC, Wetle T, Shield R, et al. Family perspectives on end-of-life care at the last place of care. JAMA J Am Med Assoc. 2004;291(1):88-93.

32. Van den Block $L$, Deschepper R, Drieskens K, Bauwens S, Bilsen J, Bossuyt N, et al. Hospitalisations at the end of life: using a sentinel surveillance network to study hospital use and associated patient, disease and healthcare factors. BMC Health Serv Res. 2007;7:69.

33. Gomes B, Calanzani N, Curiale V, Mccrone P, Higginson I. Effectiveness and cost-effectiveness of home palliative care services for adults with advanced illness and their caregivers. Cochrane Database Syst Rev. 2014;6:CD007760.

34. Miccinesi G, Crocetti E, Morino P, Fallai M, Piazza M, Cavallini V, et al. Palliative home care reduces time spent in hospital wards: a population-based study in the Tuscany Region, Italy. Cancer Causes Control. 2003;14(10):971-7.

35. Centeno C, Clark D, Lynch T, Racafort J, Praill D, De Lima L, et al. Facts and indicators on palliative care development in 52 countries of the WHO European region: results of an EAPC task force. Palliat Med. 2007;21 (6):463-71.

36. Baker M, Luce J, Bosslet GT. Integration of palliative care services in the intensive care unit. Clin Chest Med. 2015;36(3):441-8. Elsevier Inc.

37. Swiss Federal Statistical Office. Inventar der Datensituation über Palliative Care. Neuenburg: Swiss Federal Statistical Office; 2012.

38. Bayer-Oglesby L, Höpflinger F. Statistische Grundlagen zur regionalen Pflegeheimplanung in der Schweiz. Methodik und kantonale Kennzahlen. Neuenburg: Obsan; 2010

39. Fischer S, Bosshard G, Zellweger U, Faisst K. Der Sterbeort: "Wo sterben die Menschen heute in der Schweiz?". Z Gerontol Geriatr. 2004;37(6):467-74.

\section{Submit your next manuscript to BioMed Central and we will help you at every step:}

- We accept pre-submission inquiries

- Our selector tool helps you to find the most relevant journal

- We provide round the clock customer support

- Convenient online submission

- Thorough peer review

- Inclusion in PubMed and all major indexing services

- Maximum visibility for your research

Submit your manuscript at www.biomedcentral.com/submit
C Biomed Central 